\title{
A Generalized Algorithm for Solving Nonlinear Equations Using Distributions
}

\author{
Houssam Houssein ${ }^{1}$ \\ ${ }^{1}$ Independent Researcher, Avenue de l'Arche, 92400 Courbevoie, France \\ Correspondence: Houssam Houssein, Avenue de l'Arche, 92400 Courbevoie, France. Tel: +33-7-8625-4901. E-mail: \\ houssamhoussein6@gmail.com, houssam.houssein@centraliens.net
}

Received: September 4, 2018 Accepted: September 20, 2018 Online Published: October 15, 2018

doi:10.5539/jmr.v10n6p1 URL: https://doi.org/10.5539/jmr.v10n6p1

\begin{abstract}
In this paper we introduce a method for solving the nonlinear equation $f(x)=0$ where $x, f(x)$ are two vectors. This method uses the theory of distributions, and it's a non-iterative method, indeed it creates a sequence of vectors with an explicit formula and this sequence will converge to the solution of the nonlinear equation.
\end{abstract}

Keywords: Dirac, distributions, nonlinear equation, system

\section{Introduction}

In general, iterative methods are used for solving nonlinear equations $(f(x)=0)$, thus a starting point of the iterative sequence is required for these methods. The starting point must be in a neighborhood and close enough to the solution of the nonlinear equation so that the sequence converges to the solution, which is a disadvantage for these methods.

This paper is a generalization of (Houssein, 2018) in order to treat the case where $x$ and $f(x)$ are two vectors. therefore the method in this paper will produce a sequence of vectors with an explicit formula, where the solution of the nonlinear equation is the limit of this sequence. In the following we will study a sequence of distributions in order to present the algorithm responsible of the creation of this sequence which converges to the solution, in addition two examples are presented to illustrate the method.

The explicit formula of the sequence and the requirement of only few conditions for the function $f$ in a neighborhood of the solution are the advantages of this method.

1.1 Distributions and Test Functions (Houssein, 2018), (Schwartz, 1963), (Schwartz, 1966)

Let $\Omega \subseteq \mathbb{R}^{d}$ be an open set with $d \in \mathbb{N}^{*}$, and let $f$ a locally integrable function on $\Omega, f: \Omega \rightarrow \mathbb{R}$, then the considered distribution $T$ is the following:

$$
<T, \phi>=T(\phi)=\int_{\Omega} f \phi d \lambda \quad \forall \phi \in \mathcal{D}(\Omega)
$$

Where $\lambda$ is the Lebesgue measure, $\mathcal{D}(\Omega)$ the space of the test functions on $\Omega$. T is also noted by $f$, in other words $<f, \phi>=<T, \phi>$.

An example of a test function belonging to $\mathcal{D}\left(\mathbb{R}^{d}\right)$ is the following:

$$
\phi(x)=\left\{\begin{array}{l}
e^{\frac{-1}{1-\|x\|^{2}}} \quad \text { if }\|x\|<1 \\
0 \text { otherwise }
\end{array}\right.
$$

\section{Building a Sequence of Distributions That Converges to a Dirac}

In the following we consider a function $g: \bar{\Omega} \rightarrow \mathbb{R}$ where $\Omega \subset \mathbb{R}^{d}$ is a non-empty, bounded and open set with $d \in \mathbb{N}^{*}$. The function $g$ is supposed to be a positive function $g(x) \geq 0 \forall x \in \bar{\Omega}$. Using the theorem 1 we will be able to create a sequence of distributions which converges to the Dirac distribution.

Theorem 1 We suppose that the function $g$ has only one zero $x^{*}$ in $\bar{\Omega}$ and $x^{*} \in \Omega$ such that $g$ is $C^{2}$ in a neighborhood of $x^{*}$ and the Hessian matrix at $x^{*}$ : Hess $s_{x^{*}}(g)$ is positive definite, in addition we suppose that exists a neighborhood of $x^{*}$ which contains a finite number of critical points of $g$, if we consider the sequence of functions $g_{n}$ defined by: 
$g_{n}(x)=\boldsymbol{1}_{\Omega}(x)\left(n \sqrt{\frac{1}{2 \pi}}\right)^{d} e^{-n^{2} g(x)}$ then:

$$
g_{n} \underset{n \rightarrow+\infty}{\longrightarrow} \frac{1}{\sqrt{c}} \delta_{x^{*}} \quad \text { In the sense of distributions }
$$

Where $c=\operatorname{det}\left(\operatorname{Hess}_{x^{*}}(g)\right), \delta_{x^{*}}$ the Dirac distribution at $x^{*}$. The convergence in the sense of distributions means that:

$$
<g_{n}, \phi>=\int_{\mathbb{R}^{d}} g_{n}(x) \phi(x) d \lambda(x) \underset{n \rightarrow+\infty}{\longrightarrow} \frac{1}{\sqrt{c}} \phi\left(x^{*}\right) \quad \forall \phi \in \mathcal{D}\left(\mathbb{R}^{d}\right)
$$

Proof. At first because $g \geq 0$, the function $g$ reaches its minimum at $x^{*}$, thus:

$$
J a c_{x^{*}}(g)=\left.\left(\frac{\partial g}{\partial x_{1}}, \ldots, \frac{\partial g}{\partial x_{d}}\right)\right|_{x=x^{*}}=0
$$

Where $x=\left(x_{1}, \ldots, x_{d}\right)$.

Using the Taylor's theorem $g$ can be expressed by:

$$
\begin{gathered}
g(x)=g\left(x^{*}\right)+\operatorname{Jac}_{x^{*}}(g) \cdot\left(x-x^{*}\right)+\frac{1}{2}{ }^{t}\left(x-x^{*}\right) \cdot \operatorname{Hess}_{x^{*}}(g) \cdot\left(x-x^{*}\right)+\left\|x-x^{*}\right\|^{2} \epsilon\left(x-x^{*}\right) \\
\Rightarrow g(x)=\frac{1}{2}^{t}\left(x-x^{*}\right) \cdot \operatorname{Hess}_{x^{*}}(g) \cdot\left(x-x^{*}\right)+\left\|x-x^{*}\right\|^{2} \epsilon\left(x-x^{*}\right)
\end{gathered}
$$

Where $\epsilon$ is a function $\epsilon: \mathbb{R}^{d} \rightarrow \mathbb{R}$ such that: $\epsilon(x) \underset{x \rightarrow 0}{\longrightarrow} 0$ and where:

$$
\operatorname{Hess}_{x^{*}}(g)=\left(\begin{array}{ccc}
\frac{\partial^{2} g}{\partial x_{1}^{2}}\left(x^{*}\right) & \ldots & \frac{\partial^{2} g}{\partial x_{1} \partial x_{d}}\left(x^{*}\right) \\
\vdots & \ddots & \vdots \\
\frac{\partial^{2} g}{\partial x_{1} \partial x_{d}}\left(x^{*}\right) & \ldots & \frac{\partial^{2} g}{\partial x_{d}^{2}}\left(x^{*}\right)
\end{array}\right)
$$

The Hessian matrix at $x^{*}: \operatorname{Hess}_{x^{*}}(g)$ is symmetric and positive definite therefore:

$$
\operatorname{Hess}_{x^{*}}(g)={ }^{t} O D O
$$

Where $O$ is a $d \times d$ orthogonal matrix and $D$ a $d \times d$ diagonal matrix:

$$
D=\left(\begin{array}{cccc}
c_{1} & 0 & \ldots & 0 \\
0 & c_{2} & \ldots & 0 \\
\vdots & \ddots & \ddots & \vdots \\
0 & 0 & \ldots & c_{d}
\end{array}\right)
$$

Where $c_{1}, \ldots, c_{d}>0$.

We obtain that:

$$
g(x)=\frac{1}{2}{ }^{t}\left(O \cdot\left(x-x^{*}\right)\right) \cdot D \cdot\left(O \cdot\left(x-x^{*}\right)\right)+\left\|x-x^{*}\right\|^{2} \epsilon\left(x-x^{*}\right)
$$

For a $\phi \in \mathcal{D}\left(\mathbb{R}^{d}\right)$ we consider the distribution sequence:

$$
\begin{gathered}
<g_{n}, \phi>=\int_{\mathbb{R}^{d}} g_{n}(x) \phi(x) d \lambda(x) \\
\Rightarrow<g_{n}, \phi>=\int_{\mathbb{R}^{d}} \mathbf{1}_{\Omega}(x)\left(n \sqrt{\frac{1}{2 \pi}}\right)^{d} e^{-n^{2} g(x)} \phi(x) d \lambda(x) \\
\Rightarrow<g_{n}, \phi>=\int_{\mathbb{R}^{d}} \mathbf{1}_{\Omega}(x)\left(n \sqrt{\frac{1}{2 \pi}}\right)^{d} e^{-n^{2}\left(\frac{1}{2}{ }^{t}\left(O \cdot\left(x-x^{*}\right)\right) \cdot D \cdot\left(O \cdot\left(x-x^{*}\right)\right)+\left\|x-x^{*}\right\|^{2} \epsilon\left(x-x^{*}\right)\right)} \phi(x) d \lambda(x)
\end{gathered}
$$


If we make a substitution $y=x-x^{*}$ we obtain:

$$
<g_{n}, \phi>=\int_{\mathbb{R}^{d}} \mathbf{1}_{\Omega^{*}}(y)\left(n \sqrt{\frac{1}{2 \pi}}\right)^{d} e^{-n^{2}\left(\frac{1}{2} t(O . y) \cdot D \cdot(O . y)+\|y\|^{2} \epsilon(y)\right)} \phi\left(y+x^{*}\right) d \lambda(y)
$$

Where $\Omega^{*}=\left\{x-x^{*} \mid x \in \Omega\right\} . \Omega^{*}$ is a neighborhood of 0 .

Another substitution is made $x=O y$ thus we obtain:

$$
\mathbf{1}_{\Omega^{*}}(y)=\mathbf{1}_{\Omega^{*}}\left(O^{-1} x\right)=\mathbf{1}_{O \Omega^{*}}(x)
$$

Where $O \Omega^{*}=\left\{O . y \mid y \in \Omega^{*}\right\}, O \Omega^{*}$ is also a neighborhood of 0 , indeed because $\Omega^{*}$ is a a neighborhood of 0 then there exist a ball $B(0, r)=\left\{x \in \mathbb{R}^{d} \mid\|x\|<r\right\}$ such that $B(0, r) \subset \Omega^{*} . O(B(0, r)) \subset O \Omega^{*}$ because $O$ is bijective and:

$$
O(B(0, r))=\left\{O . x \mid x \in \mathbb{R}^{d} \&\|x\|<r\right\}
$$

In addition we have

$$
\|O x\|^{2}={ }^{t}(O \cdot x) \cdot(O x)={ }^{t} x\left({ }^{t} O \cdot O\right) \cdot x={ }^{t} x \cdot I d \cdot x={ }^{t} x \cdot x=\|x\|^{2}
$$

Then $\|O x\|=\|x\|$ and

$$
\Rightarrow O(B(0, r))=\left\{O . x \mid x \in \mathbb{R}^{d} \&\|x\|<r\right\} \subset B(0, r)=\left\{y \in \mathbb{R}^{d} \mid\|y\|<r\right\}
$$

In the other sens let $y \in \mathbb{R}^{d}$ such that $\|y\|<r$ then $y=O .\left(O^{-1} . y\right)$ thus $y \in O(B(0, r))$ and $B(0, r) \subset O(B(0, r))$.

We deduce that $B(0, r)=O(B(0, r)) \subset O \Omega^{*}$ therefore $O \Omega^{*}$ is a neighborhood of 0 .

Back to the substitution $x=O y$, the Jacobian matrix of this transforamtion is equal to $J a c=O^{-1}$, therefore $|\operatorname{det}(\mathrm{Jac})|=1$. We know also that $O^{-1}\left(\mathbb{R}^{d}\right)=\mathbb{R}^{d}$ because $O^{-1}$ is bijective. Therefore:

$$
<g_{n}, \phi>=\int_{\mathbb{R}^{d}} \mathbf{1}_{O \Omega^{*}}(x)\left(n \sqrt{\frac{1}{2 \pi}}\right)^{d} e^{-n^{2}\left(\frac{1}{2}{ }^{t} x \cdot D \cdot x+\|x\|^{2} \epsilon\left(O^{-1} . x\right)\right)} \phi\left(O^{-1} . x+x^{*}\right) d \lambda(x)
$$

Let the sequence $E_{n}$ be defined by:

$$
<E_{n}, \phi>=\int_{\mathbb{R}^{d}}\left(n \sqrt{\frac{1}{2 \pi}}\right)^{d} e^{-\frac{1}{2} n^{2}\left({ }^{t} x \cdot D \cdot x\right)} \phi\left(O^{-1} \cdot x+x^{*}\right) d \lambda(x)
$$

we will prove next that:

$$
<E_{n}, \phi>\underset{n \rightarrow+\infty}{\longrightarrow} \frac{1}{\sqrt{\operatorname{det}\left(\operatorname{Hess}_{x^{*}}(g)\right)}} \phi\left(x^{*}\right)
$$

Indeed

$$
\begin{aligned}
{ }^{t} x . D . x & =\left(x_{1}, \ldots, x_{d}\right) \cdot\left(\begin{array}{cccc}
c_{1} & 0 & \ldots & 0 \\
0 & c_{2} & \ldots & 0 \\
\vdots & \ddots & \ddots & \vdots \\
0 & 0 & \ldots & c_{d}
\end{array}\right) \cdot\left(\begin{array}{c}
x_{1} \\
\vdots \\
x_{d}
\end{array}\right) \\
& \Rightarrow{ }^{t} x . D . x=c_{1} x_{1}^{2}+\ldots+c_{d} x_{d}^{2}
\end{aligned}
$$

Let $I_{n}=\int_{\mathbb{R}^{d}}\left(n \sqrt{\frac{1}{2 \pi}}\right)^{d} e^{-\frac{1}{2} n^{2}\left({ }^{t} x \cdot D \cdot x\right)} d \lambda(x)$ then:

$$
I_{n}=\int_{\mathbb{R}^{d}}\left(n \sqrt{\frac{1}{2 \pi}}\right)^{d} e^{-\frac{1}{2} n^{2}\left(c_{1} x_{1}^{2}+\ldots+c_{d} x_{d}^{2}\right)} d \lambda(x)
$$

Let the substitution be $y=n x \Longleftrightarrow\left(y_{1}, \ldots, y_{d}\right)=n\left(x_{1}, \ldots, x_{d}\right)$ then the Jacobian matrix of this transformation is equal to $J a c=\frac{1}{n} I_{d}$ and $|\operatorname{det}(J a c)|=\left(\frac{1}{n}\right)^{d}$. We will obtain:

$$
I_{n}=\left(\sqrt{\frac{1}{2 \pi}}\right)^{d} \int_{\mathbb{R}^{d}} e^{-\frac{1}{2}\left(c_{1} y_{1}^{2}+\ldots+c_{d} y_{d}^{2}\right)} d \lambda(y)
$$


Using Fubini's theorem:

$$
\begin{aligned}
& I_{n}=\left(\sqrt{\frac{1}{2 \pi}}\right) \int_{\mathbb{R}^{d}} e^{-\frac{1}{2} c_{1} y_{1}^{2}} d \lambda\left(y_{1}\right) \times \ldots \times\left(\sqrt{\frac{1}{2 \pi}}\right) \int_{\mathbb{R}^{d}} e^{-\frac{1}{2} c_{d} y_{d}^{2}} d \lambda\left(y_{d}\right) \\
& \Rightarrow I_{n}=\frac{1}{\sqrt{c_{1} \times \ldots \times c_{d}}}=\frac{1}{\sqrt{\operatorname{det}\left(\operatorname{Hess}_{x^{*}}(g)\right)}}
\end{aligned}
$$

Using the substitution $y=n x$ for $E_{n}$ :

$$
<E_{n}, \phi>=\left(\sqrt{\frac{1}{2 \pi}}\right)^{d} \int_{\mathbb{R}^{d}} e^{-\frac{1}{2}\left(c_{1} y_{1}^{2}+\ldots+c_{d} y_{d}^{2}\right)} \phi\left(O^{-1} \cdot \frac{y}{n}+x^{*}\right) d \lambda(y)
$$

Otherwise we have:

$$
\begin{gathered}
\forall y \in \mathbb{R}^{d}, \forall n \in \mathbb{N}^{*},\left|\phi\left(O^{-1} \cdot \frac{y}{n}+x^{*}\right)\right| \leq \sup _{y \in \mathbb{R}^{d}}(|\phi(y)|)=M<\infty \\
\Rightarrow \forall y \in \mathbb{R}^{d}, \forall n \in \mathbb{N}^{*},\left|e^{-\frac{1}{2}\left(c_{1} y_{1}^{2}+\ldots+c_{d} y_{d}^{2}\right)} \cdot \phi\left(O^{-1} \cdot \frac{y}{n}+x^{*}\right)\right| \leq M \cdot e^{-\frac{1}{2}\left(c_{1} y_{1}^{2}+\ldots+c_{d} y_{d}^{2}\right)}
\end{gathered}
$$

The application $e^{-\frac{1}{2}\left(c_{1} y_{1}^{2}+\ldots+c_{d} y_{d}^{2}\right)} \cdot \phi\left(O^{-1} \cdot \frac{y}{n}+x^{*}\right)$ is measurable and the application $e^{-\frac{1}{2}\left(c_{1} y_{1}^{2}+\ldots+c_{d} y_{d}^{2}\right)}$ is integrable, therefore using the Dominated Convergence theorem we deduce that:

$$
\begin{aligned}
<E_{n}, \phi & >\underset{n \rightarrow+\infty}{\longrightarrow}\left(\sqrt{\frac{1}{2 \pi}}\right)^{d} \int_{\mathbb{R}^{d}} e^{-\frac{1}{2}\left(c_{1} y_{1}^{2}+\ldots+c_{d} y_{d}^{2}\right)} d \lambda(y) . \phi\left(x^{*}\right) \\
& \Rightarrow<E_{n}, \phi>\underset{n \rightarrow+\infty}{\longrightarrow} \frac{1}{\sqrt{\operatorname{det}\left(\operatorname{Hess}_{x^{*}}(g)\right)}} \phi\left(x^{*}\right)
\end{aligned}
$$

Let $G_{n}$ and $F_{n}$ be two sequence of functions defined on $\mathbb{R}^{d}$ by: $G_{n}(x)=\mathbf{1}_{O \Omega^{*}}(x)\left(n \sqrt{\frac{1}{2 \pi}}\right)^{d} e^{-n^{2}\left(\frac{1}{2} t^{t} x \cdot D \cdot x+\|x\|^{2} \epsilon\left(O^{-1} . x\right)\right)}$ and $F_{n}(x)=\left(n \sqrt{\frac{1}{2 \pi}}\right)^{d} e^{-\frac{1}{2} n^{2}\left({ }^{t} x \cdot D \cdot x\right)}$.

We will prove next that:

$$
\left\|G_{n}-F_{n}\right\|_{L^{1}\left(\mathbb{R}^{d}\right)} \underset{n \rightarrow+\infty}{\longrightarrow} 0
$$

First we will study it on the ball $B\left(0, \frac{1}{\sqrt{n}}\right)$.

There $\exists N_{1} \in \mathbb{N}^{*}$ such that $B\left(0, \frac{1}{\sqrt{n}}\right) \subset O \Omega^{*} \forall n \geq N_{1}$ because $O \Omega^{*}$ is a neighborhood of $0_{\mathbb{R}^{d}}$. Therefore:

$$
\int_{B\left(0, \frac{1}{\sqrt{n}}\right)}\left|G_{n}-F_{n}\right| d \lambda=\left(n \sqrt{\frac{1}{2 \pi}}\right)^{d} \int_{B\left(0, \frac{1}{\sqrt{n}}\right)} e^{-\frac{1}{2} n^{2}\left(t^{t} x \cdot D \cdot x\right)}\left|1-e^{-n^{2}\|x\|^{2} \epsilon\left(O^{-1} x\right)}\right| d \lambda(x)
$$

By making the substitution $y=n x$ :

$$
\int_{B\left(0, \frac{1}{\sqrt{n}}\right)}\left|G_{n}-F_{n}\right| d \lambda=\left(\sqrt{\frac{1}{2 \pi}}\right)^{d} \int_{B(0, \sqrt{n})} e^{-\frac{1}{2}\left(c_{1} y_{1}^{2}+\ldots+c_{d} y_{d}^{2}\right)}\left|1-e^{-\|y\|^{2} \epsilon\left(O^{-1} \frac{y}{n}\right)}\right| d \lambda(y)
$$

Besides we have $\epsilon(y) \underset{y \rightarrow 0}{\longrightarrow} 0$ and:

$$
\left\|O^{-1} \frac{y}{n}\right\|^{2}={ }^{t}\left(O^{-1} \frac{y}{n}\right) \cdot\left(O^{-1} \frac{y}{n}\right)={ }^{t}\left(\frac{y}{n}\right) \cdot O \cdot O^{-1} \cdot \frac{y}{n}=\frac{1}{n^{2}}\|y\|^{2}
$$

Because $O^{-1}={ }^{t} O$.

Let $0<\eta<\frac{1}{2} \operatorname{Min}\left(c_{1}, \ldots, c_{d}\right)$ then:

$$
\exists A>0, \forall y \in \mathbb{R}^{d} \text { such that }\|y\| \leq A, \Rightarrow|\epsilon(y)|<\eta
$$


We have $\|y\|<\sqrt{n} \Rightarrow \frac{1}{n}\|y\| \leq \frac{1}{\sqrt{n}}$.

There $\exists N_{2} \in \mathbb{N}^{*}, \forall n \geq N_{2} \Rightarrow \frac{1}{\sqrt{n}}<A$ thus:

$$
\begin{gathered}
\left\|O^{-1} \frac{y}{n}\right\|<A \quad \forall n \geq N_{3}=\operatorname{Max}\left(N_{1}, N_{2}\right) \\
\Rightarrow\left|\epsilon\left(O^{-1} \frac{y}{n}\right)\right|<\eta \quad \forall n \geq N_{3}
\end{gathered}
$$

For $n \geq N_{3}$ we have two cases to treat:

1. If $0 \leq \epsilon\left(O^{-1} \frac{y}{n}\right) \leq \eta \Rightarrow 1-e^{-\|y\|^{2} \epsilon\left(O^{-1} \frac{y}{n}\right)} \leq 1-e^{-\|y\|^{2} \eta}$, and because $\|y\|^{2} \epsilon\left(O^{-1} \frac{y}{n}\right) \geq 0$ :

$$
\left|1-e^{-\|y\|^{2} \epsilon\left(O^{\left.-1 \frac{y}{n}\right)}\right.}\right|=1-e^{-\|y\|^{2} \epsilon\left(O^{-1} \frac{y}{n}\right)} \leq 1-e^{-\|y\|^{2} \eta}
$$

2. If $-\eta \leq \epsilon\left(O^{-1} \frac{y}{n}\right) \leq 0 \Rightarrow e^{-\|y\|^{2} \epsilon\left(O^{-1} \frac{y}{n}\right)}-1 \leq e^{\|y\|^{2} \eta}-1$, and because $-\|y\|^{2} \epsilon\left(O^{-1} \frac{y}{n}\right) \geq 0$ :

$$
\left|1-e^{-\|y\|^{2} \epsilon\left(O^{-1} \frac{y}{n}\right)}\right|=e^{-\|y\|^{2} \epsilon\left(O^{-1} \frac{y}{n}\right)}-1 \leq e^{\|y\|^{2} \eta}-1
$$

We conclude that:

$$
\begin{gathered}
\forall n \geq N_{3} \Rightarrow\left|1-e^{-\|y\|^{2} \epsilon\left(O^{-1} \frac{y}{n}\right)}\right| \leq \operatorname{Max}\left(1-e^{-\|y\|^{2} \eta}, e^{\|y\|^{2} \eta}-1\right) \leq\left(1-e^{-\|y\|^{2} \eta}\right)+\left(e^{\|y\|^{2} \eta}-1\right) \\
\Rightarrow \forall n \geq N_{3} \quad\left|1-e^{-\|y\|^{2} \epsilon\left(O^{-1} \frac{y}{n}\right)}\right| \leq e^{\eta\|y\|^{2}}-e^{-\eta\|y\|^{2}}
\end{gathered}
$$

We deduce that $\forall n \geq N_{3}$ :

$$
\begin{gathered}
\int_{B\left(0, \frac{1}{\sqrt{n}}\right)}\left|G_{n}-F_{n}\right| d \lambda \leq\left(\sqrt{\frac{1}{2 \pi}}\right)^{d} \int_{\mathbb{R}^{d}} e^{-\frac{1}{2}\left(c_{1} y_{1}^{2}+\ldots+c_{d} y_{d}^{2}\right)} \cdot\left(e^{\eta\|y\|^{2}}-e^{-\eta\|y\|^{2}}\right) d \lambda(y) \\
e^{-\frac{1}{2}\left(c_{1} y_{1}^{2}+\ldots+c_{d} y_{d}^{2}\right)} \cdot\left(e^{\eta\|y\|^{2}}-e^{-\eta\|y\|^{2}}\right)=e^{-\frac{1}{2}\left(\left(c_{1}-2 \eta\right) y_{1}^{2}+\ldots+\left(c_{d}-2 \eta\right) y_{d}^{2}\right)}-e^{-\frac{1}{2}\left(\left(c_{1}+2 \eta\right) y_{1}^{2}+\ldots+\left(c_{d}+2 \eta\right) y_{d}^{2}\right)}
\end{gathered}
$$

By supposition $\eta<\frac{1}{2} \operatorname{Min}\left(c_{1}, \ldots, c_{d}\right)$ then:

$$
c_{i}-2 \eta>0 \quad c_{i}+2 \eta>0 \quad \forall 1 \leq i \leq d
$$

Therefore:

$$
\int_{\mathbb{R}^{d}} e^{-\frac{1}{2}\left(\left(c_{1}-2 \eta\right) y_{1}^{2}+\ldots+\left(c_{d}-2 \eta\right) y_{d}^{2}\right)} d \lambda(y)<\infty \text { and } \int_{\mathbb{R}^{d}} e^{-\frac{1}{2}\left(\left(c_{1}+2 \eta\right) y_{1}^{2}+\ldots+\left(c_{d}+2 \eta\right) y_{d}^{2}\right)} d \lambda(y)<\infty
$$

In the other hand for $y$ fixed:

$$
\mathbf{1}_{B(0, \sqrt{n})}(y) e^{-\frac{1}{2}\left(c_{1} y_{1}^{2}+\ldots+c_{d} y_{d}^{2}\right)} \mid 1-e^{-\|y\|^{2} \epsilon\left(O^{-1} \frac{y}{n}\right)} \underset{n \rightarrow+\infty}{\longrightarrow} 0
$$

Using the Dominated Convergence theorem, we deduce that:

$$
\int_{B\left(0, \frac{1}{\sqrt{n}}\right)}\left|G_{n}-F_{n}\right| d \lambda \underset{n \rightarrow+\infty}{\longrightarrow} 0
$$

The second part of the proof will use the lemma 1 presented after the proof. The lemma 1 says that there exists a $R>0$ where

$$
\forall 0<r<R, \exists \zeta \in C\left(x^{*}, r\right) \text { such that } g(\zeta) \leq g(x) \forall x \in \mathbb{R}^{d} \& r<\left\|x-x^{*}\right\| \leq R
$$

Where $C\left(x^{*}, r\right)=\left\{x \in \mathbb{R}^{d} \mid\left\|x-x^{*}\right\|=r\right\}, R$ is chosen such that $\bar{B}\left(x^{*}, R\right) \subset \Omega$.

Besides $\exists N_{4}>N_{3}$ such that $\forall n \in \mathbb{N}$ and $n>N_{4} \Rightarrow \frac{1}{\sqrt{n}}<R$ thus:

$$
\forall n>N_{4}, \exists \zeta_{n} \in C\left(x^{*}, \frac{1}{\sqrt{n}}\right) \text { such that } g\left(\zeta_{n}\right) \leq g(x) \forall x \in C\left(x^{*}, \frac{1}{\sqrt{n}}, R\right)
$$

Where $C\left(x^{*}, \frac{1}{\sqrt{n}}, R\right)=\left\{x \in \mathbb{R}^{d} \mid \frac{1}{\sqrt{n}}<\left\|x-x^{*}\right\| \leq R\right\}$

By making two substitutions $y=x-x^{*}$ and then $x=O . y$ we obtain:

$$
\int_{C\left(x^{*}, \frac{1}{\sqrt{n}}, R\right)} g_{n} d \lambda=\int_{C\left(0, \frac{1}{\sqrt{n}}, R\right)} G_{n} d \lambda
$$


Thus

$$
\int_{C\left(0, \frac{1}{\sqrt{n}}, R\right)} G_{n} d \lambda \leq \lambda\left(B\left(x^{*}, R\right)\right) \cdot\left(n \sqrt{\frac{1}{2 \pi}}\right)^{d} e^{-n^{2} g\left(\zeta_{n}\right)}
$$

Where $\lambda\left(B\left(x^{*}, R\right)\right)<\infty$ and $\zeta_{n} \in C\left(x^{*}, \frac{1}{\sqrt{n}}\right)$.

$$
e^{-n^{2} g\left(\zeta_{n}\right)}=e^{-n^{2}\left(\frac{1}{2} y_{n} \cdot D \cdot y_{n}+\left\|O^{-1} \cdot y_{n}\right\|^{2} \epsilon\left(O^{-1} \cdot y_{n}\right)\right)}
$$

Where $y_{n}=O \cdot\left(\zeta_{n}-x^{*}\right)$

$$
\left\|O^{-1} \cdot y_{n}\right\|=\left\|\zeta_{n}-x^{*}\right\|=\frac{1}{\sqrt{n}}
$$

And

$$
{ }^{t} y_{n} \cdot D \cdot y_{n}=c_{1} y_{n 1}^{2}+\ldots+c_{d} y_{n d}^{2}
$$

Let $k=\min _{1 \leq i \leq d}\left(c_{i}\right)$ then:

$$
{ }^{t} y_{n} \cdot D \cdot y_{n} \geq k\left(y_{n 1}^{2}+\ldots+y_{n d}^{2}\right)=k\left\|y_{n}\right\|^{2}=k \frac{1}{n}
$$

Therefore

$$
e^{-n^{2} g\left(\zeta_{n}\right)} \leq e^{-\frac{1}{2} n k-n \epsilon\left(O^{-1} \cdot y_{n}\right)}=e^{-\frac{1}{2} n k} \cdot e^{-n \epsilon\left(O^{-1} \cdot y_{n}\right)}
$$

Otherwise let $0<\eta<\frac{1}{2} k$ then:

$$
\exists A>0, \forall y \in \mathbb{R}^{d} \text { such that }\|y\| \leq A, \Rightarrow|\epsilon(y)|<\eta
$$

We have $\left\|O^{-1} \cdot y_{n}\right\| \leq \frac{1}{\sqrt{n}}$.

There $\exists N_{5}>N_{4} \in \mathbb{N}^{*}, \forall n \geq N_{5} \Rightarrow \frac{1}{\sqrt{n}}<A$ thus:

$$
\begin{gathered}
\left\|O^{-1} \cdot y_{n}\right\|<A \quad \forall n \geq N_{5} \\
\Rightarrow\left|\epsilon\left(O^{-1} \cdot y_{n}\right)\right|<\eta \quad \forall n \geq N_{5}
\end{gathered}
$$

For $n \geq N_{5}$ we have two cases to treat:

1. If $0 \leq \epsilon\left(O^{-1} \cdot y_{n}\right) \leq \eta \Rightarrow e^{-n \epsilon\left(O^{-1} \cdot y_{n}\right)} \leq 1$

2. If $-\eta \leq \epsilon\left(O^{-1} \cdot y_{n}\right) \leq 0 \Rightarrow e^{-n \epsilon\left(O^{-1} \cdot y_{n}\right)} \leq e^{n \eta}$

Thus $e^{-n \epsilon\left(O^{-1} \cdot y_{n}\right)} \leq 1+e^{n \eta}$ and

$$
e^{-n^{2} g\left(\zeta \zeta_{n}\right)} \leq e^{-\frac{1}{2} n k}+e^{-n\left(\frac{1}{2} k-\eta\right)}
$$

By supposition $\frac{1}{2} k-\eta>0$ Therefore

$$
\int_{C\left(0, \frac{1}{\sqrt{n}}, R\right)} G_{n} d \lambda \underset{n \rightarrow+\infty}{\longrightarrow} 0
$$

In the other hand $F_{n}(x)=\left(n \sqrt{\frac{1}{2 \pi}}\right)^{d} e^{-\frac{1}{2} n^{2}\left({ }^{t} x \cdot D \cdot x\right)}$

$$
\begin{gathered}
F_{n}(x) \leq\left(n \sqrt{\frac{1}{2 \pi}}\right)^{d} e^{-\frac{1}{2} n^{2}\left(k\|x\|^{2}\right)} \\
\Rightarrow \int_{C\left(0, \frac{1}{\sqrt{n}}, R\right)} F_{n} d \lambda \leq \lambda(B(0, R)) \cdot\left(n \sqrt{\frac{1}{2 \pi}}\right)^{d} e^{-\frac{1}{2} n^{2}\left(k \frac{1}{n}\right)} \underset{n \rightarrow+\infty}{\longrightarrow} 0
\end{gathered}
$$

Therefore

$$
\int_{C\left(0, \frac{1}{\sqrt{n}}, R\right)}\left|G_{n}-F_{n}\right| d \lambda \leq \int_{C\left(0, \frac{1}{\sqrt{n}}, R\right)} G_{n} d \lambda+\int_{C\left(0, \frac{1}{\sqrt{n}}, R\right)} F_{n} d \lambda \underset{n \rightarrow+\infty}{\longrightarrow} 0
$$

We deduce that:

$$
\int_{B(0, R)}\left|G_{n}-F_{n}\right| d \lambda \underset{n \rightarrow+\infty}{\longrightarrow} 0
$$


Finally on $\bar{\Omega} \backslash B\left(x^{*}, R\right)$ the application $g$ is strictly positive $g>0$ then $g$ reaches its minimum at $\sigma \in \bar{\Omega}$ where $g(\sigma)>0$.

Besides we have:

$$
\int_{\mathbb{R}^{d}} G_{n} d \lambda=\int_{\mathbb{R}^{d}} g_{n} d \lambda \text { and } \int_{B(0, R)} G_{n} d \lambda=\int_{B\left(x^{*}, R\right)} g_{n} d \lambda
$$

Therefore

$$
\int_{\mathbb{R}^{d} \backslash B(0, R)} G_{n} d \lambda=\int_{\mathbb{R}^{d} \backslash B\left(x^{*}, R\right)} g_{n} d \lambda=\left(n \sqrt{\frac{1}{2 \pi}}\right)^{d} \int_{\Omega \backslash B\left(x^{*}, R\right)} e^{-n^{2} g(x)} d \lambda(x)
$$

And

$$
\int_{\mathbb{R}^{d} \backslash B(0, R)} G_{n} d \lambda \leq\left(n \sqrt{\frac{1}{2 \pi}}\right)^{d} \cdot \lambda\left(\Omega \backslash B\left(x^{*}, R\right)\right) \cdot e^{-n^{2} g(\sigma)} \underset{n \rightarrow+\infty}{\longrightarrow} 0
$$

Otherwise

$$
\int_{\mathbb{R}^{d} \backslash B(0, R)} F_{n} d \lambda=\int_{\mathbb{R}^{d}} \mathbf{1}_{\{\|x\| \geq R\}}(x)\left(n \sqrt{\frac{1}{2 \pi}}\right)^{d} e^{-\frac{1}{2} n^{2}\left({ }^{t} x \cdot D \cdot x\right)} d \lambda(x)
$$

Using the substitution $y=n x$ :

$$
\int_{\mathbb{R}^{d} \backslash B(0, R)} F_{n} d \lambda=\int_{\mathbb{R}^{d}} \mathbf{1}_{\{\|y\| \geq n R\}}(y)\left(\sqrt{\frac{1}{2 \pi}}\right)^{d} e^{-\frac{1}{2}\left({ }^{t} y \cdot D \cdot y\right)} d \lambda(y)
$$

As

$$
\mathbf{1}_{\{\|y\| \geq n R\}}(y)\left(\sqrt{\frac{1}{2 \pi}}\right)^{d} e^{-\frac{1}{2}\left({ }^{t} y \cdot D \cdot y\right)} \underset{n \rightarrow+\infty}{\longrightarrow} 0 \quad \text { almost everywhere }
$$

Then

$$
\int_{\mathbb{R}^{d} \backslash B(0, R)} F_{n} d \lambda \underset{n \rightarrow+\infty}{\longrightarrow} 0
$$

And

$$
\int_{\mathbb{R}^{d} \backslash B(0, R)}\left|G_{n}-F_{n}\right| d \lambda \underset{n \rightarrow+\infty}{\longrightarrow} 0
$$

We conclude that:

$$
\left\|G_{n}-F_{n}\right\|_{L^{1}\left(\mathbb{R}^{d}\right)}=\int_{\mathbb{R}^{d}}\left|G_{n}-F_{n}\right| d \lambda \underset{n \rightarrow+\infty}{\longrightarrow} 0
$$

Finally $\forall \phi \in \mathcal{D}\left(\mathbb{R}^{d}\right)$

$$
<g_{n}, \phi>=\int_{\mathbb{R}^{d}}\left(G_{n}(x)-F_{n}(x)\right) \phi\left(O^{-1} x+x^{*}\right) d \lambda(x)+\int_{\mathbb{R}^{d}} F_{n}(x) \phi\left(O^{-1} x+x^{*}\right) d \lambda(x)
$$

As $\phi$ is bounded then :

$$
<g_{n}, \phi>\underset{n \rightarrow+\infty}{\longrightarrow} \frac{1}{\sqrt{\operatorname{det}\left(\operatorname{Hess}_{x^{*}}(g)\right)}} \phi\left(x^{*}\right)
$$

The following lemma is used in the proof of the theorem 1.

Lemma 1 Let $g$ be a positive application $g: \mathbb{R}^{d} \rightarrow \mathbb{R}_{+}$, we suppose that there exists $x^{*} \in \mathbb{R}^{d}$ such that $g\left(x^{*}\right)=0$, and that exists a neighborhood $\Omega$ of $x^{*}$ such that $g \in C^{1}(\Omega)$ and such that the number of critical points of $g$ in $\Omega$ is finite. Then

$$
\exists R>0, \forall 0<r<R, \exists \zeta \in C\left(x^{*}, r\right) \text { such that } g(\zeta) \leq g(x) \forall x \in \mathbb{R}^{d} \& r<\left\|x-x^{*}\right\| \leq R
$$

Where $C\left(x^{*}, r\right)=\left\{x \in \mathbb{R}^{d} \mid\left\|x-x^{*}\right\|=r\right\}$

Proof. We will proceed by contradiction, we will suppose that it's not true thus we have:

$$
\forall R>0, \exists 0<r<R, \forall \zeta \in C\left(x^{*}, r\right), \exists x \in \mathbb{R}^{d} \text { such that } r<\left\|x-x^{*}\right\| \leq R \& g(\zeta)>g(x)
$$

we will construct a sequence, indeed let $R_{n}>0$ for $n \in \mathbb{N}$ thus:

$$
\exists 0<r_{n}<R_{n}, \forall \zeta \in C\left(x^{*}, r_{n}\right), \exists x \in \mathbb{R}^{d} \text { such that }
$$




$$
r_{n}<\left\|x-x^{*}\right\| \leq R_{n} \& g(\zeta)>g(x)
$$

First let $R_{n}^{*}>0$ such that $B\left(x^{*}, R_{n}^{*}\right) \subset \Omega$ and $M_{n}=\inf _{x \in C\left(x^{*}, R_{n}^{*}\right)}(g(x))>0$

The application $g$ is continuous at $x^{*}$ :

$$
\forall \epsilon>0 \exists r>0, \forall x \in B\left(x^{*}, r\right) \Rightarrow\left|g(x)-g\left(x^{*}\right)\right|<\epsilon
$$

$g \geq 0$ and $g\left(x^{*}\right)=0$ then

$$
\forall \epsilon>0 \exists r>0, \forall x \in B\left(x^{*}, r\right) \Rightarrow g(x)<\epsilon
$$

Let $\epsilon_{n}=M_{n}^{*}<M_{n}$ then $\exists r_{n}^{*}>0, \forall x \in B\left(x^{*}, r_{n}^{*}\right) \Rightarrow g(x)<M_{n}^{*}<M_{n}$, we choose $r_{n}^{*}$ such that $r_{n}^{*}<R_{n}^{*}$.

Let $R_{n}<r_{n}^{*}$ then

$$
\begin{gathered}
\exists 0<r_{n}<R_{n}, \forall \zeta \in C\left(x^{*}, r_{n}\right), \exists x \in \mathbb{R}^{d} \text { such that } \\
r_{n}<\left\|x-x^{*}\right\| \leq R_{n} \& g(\zeta)>g(x)
\end{gathered}
$$

Let $\zeta_{n}$ such that $g\left(\zeta_{n}\right)=\inf _{x \in C\left(x^{*}, r_{n}\right)}(g(x))$ therefore $\exists x_{n}$ such that $r_{n}<\left\|x_{n}-x^{*}\right\| \leq R_{n}$ and

$$
g\left(x_{n}\right)<g\left(\zeta_{n}\right) \leq g(\zeta) \forall \zeta \in C\left(x^{*}, r_{n}\right)
$$

Therefore we conclude that there exists $x_{n} \in B\left(x^{*}, R_{n}^{*}\right) \backslash \bar{B}\left(x^{*}, r_{n}\right)$ such that:

$$
\left\{\begin{array}{l}
g\left(x_{n}\right)<g(\zeta) \forall \zeta \in C\left(x^{*}, r_{n}\right) \\
g\left(x_{n}\right)<M_{n}^{*}<g(y) \quad \forall y \in C\left(x^{*}, R_{n}^{*}\right)
\end{array}\right.
$$

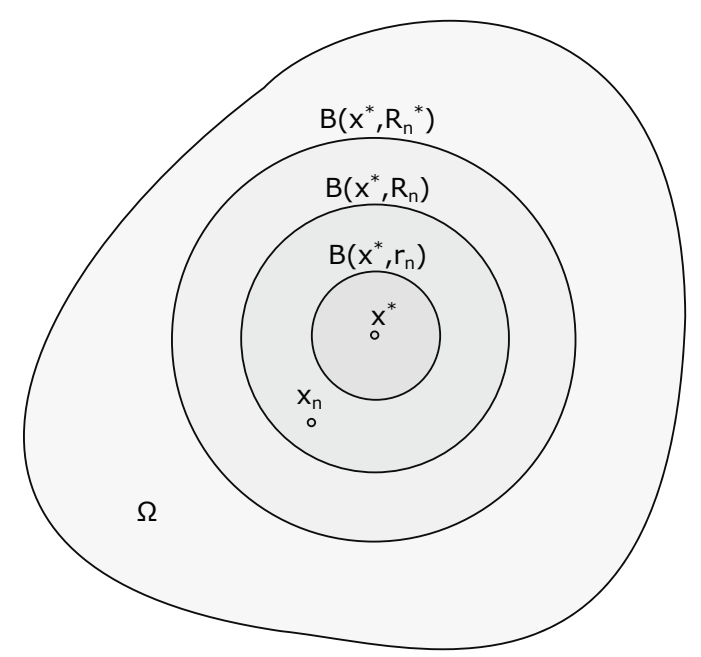

Figure 1. The neighborhood $\Omega$

Otherwise the set $O_{n}=\left\{x \in \mathbb{R}^{d} \mid r_{n} \leq\left\|x-x^{*}\right\| \leq R_{n}^{*}\right\}$ is closed and bounded thus $O_{n}$ is a compact set.

The application $g$ is continuous on $O_{n}$ thus the minimum of $g$ is reached on $O_{n}$, let $x_{n}^{*}$ denotes the vector where $g$ reaches its minimum on $O_{n} . x_{n}^{*}$ does not belong to $\partial O_{n}$ because as we saw above, $\exists x_{n}$ belongs to the interior of $O_{n}$ and $g\left(x_{n}\right)<g(y) \forall y \in \partial O_{n}$. Therefore we deduce that $x_{n}^{*}$ is a critical point.

Second we will repeat the process for the step $n+1$ by taking $R_{n+1}^{*}$ such that $R_{n+1}^{*}<\left\|x_{n}^{*}-x^{*}\right\|$, therefore we will obtain an infinity of critical points in $\Omega$, which leads to a contradiction.

In general we have the following theorem if $g$ has several zeros in $\Omega$ :

Theorem 2 If we suppose that the function $g$ has only $k$ zeros $x_{1}^{*}, \ldots, x_{k}^{*}$ in $\bar{\Omega}$ and $x_{1}^{*}, \ldots, x_{k}^{*} \in \Omega$ such that $g$ satisfies the hypotheses of the theorem 1 at each $x_{i}^{*}$, then by considering the sequence of functions $g_{n}$ defined by: $g_{n}(x)=$ 
$\mathbf{1}_{\Omega}(x)\left(n \sqrt{\frac{1}{2 \pi}}\right)^{d} e^{-n^{2} g(x)}$ we have:

$$
g_{n} \underset{n \rightarrow+\infty}{\longrightarrow} \sum_{i=1}^{k} \frac{1}{\sqrt{\operatorname{det}\left(\operatorname{Hess}_{x_{i}^{*}}(g)\right)}} \delta_{x_{i}^{*}} \quad \text { In the sense of distributions }
$$

Proof. It's enough to say that $\Omega=\bigcup_{i=1}^{k} \Omega_{i}$ and $g_{n}(x)=\sum_{i=1}^{k} \mathbf{1}_{\Omega_{i}}(x)\left(n \sqrt{\frac{1}{2 \pi}}\right)^{d} e^{-n^{2} g(x)}$ where $\Omega_{i}$ are disjoints and $x_{i}^{*} \in \stackrel{\circ}{\Omega_{i}}$.

\section{Application}

Let $(S)$ be a system of equations defined by:

$$
(S):\left\{\begin{array}{c}
f_{1}\left(x_{1}, \ldots, x_{d}\right)=0 \\
\vdots \\
f_{d}\left(x_{1}, \ldots, x_{d}\right)=0
\end{array}\right.
$$

Where $\forall i=1, \ldots, d$ the application $f_{i}: \bar{\Omega} \rightarrow \mathbb{R}$ is a function defined on $\bar{\Omega}$ where $\Omega \subset \mathbb{R}^{d}$ an open and bounded set with $d \in \mathbb{N}^{*}$. In addition we suppose that there exists only one zero $x^{*}$ of the system of equations in $\bar{\Omega}$ and $x^{*} \in \Omega$, we suppose also that $\forall i=1, \ldots, d$ the application $f_{i}$ is $C^{2}$ in a neighborhood of $x^{*}$.

Let $g$ the application $g: \bar{\Omega} \rightarrow \mathbb{R}$ defined by $g(x)=f_{1}^{2}(x)+\ldots+f_{d}^{2}(x) \forall x \in \bar{\Omega}$, we suppose that exists a neighborhood of $x^{*}$ which contains a finite number of critical points of $g$, let $g_{n}$ be a sequence of functions defined by $g_{n}(x)=\mathbf{1}_{\Omega}(x)\left(n \sqrt{\frac{1}{2 \pi}}\right)^{d} e^{-n^{2} g(x)}$ and let

$$
K=\operatorname{det}\left(2\left[{ }^{t} J a c_{x^{*}}\left(f_{1}\right) \cdot J a c_{x^{*}}\left(f_{1}\right)+\ldots+{ }^{t} J a c_{x^{*}}\left(f_{d}\right) . J a c_{x^{*}}\left(f_{d}\right)\right]\right)
$$

Where $J a c_{x^{*}}\left(f_{i}\right)$ denotes the Jacobian matrix of the application $f_{i}$.

Let $J a c_{x^{*}}(S)$ be the Jacobian of the system $(S)$ at $x^{*}$ in other words:

$$
J a c_{x^{*}}(S)=\left(\begin{array}{ccc}
\frac{\partial f_{1}}{\partial x_{1}}\left(x^{*}\right) & \ldots & \frac{\partial f_{1}}{\partial x_{d}}\left(x^{*}\right) \\
\vdots & \ddots & \vdots \\
\frac{\partial f_{d}}{\partial x_{1}}\left(x^{*}\right) & \ldots & \frac{\partial f_{d}}{\partial x_{d}}\left(x^{*}\right)
\end{array}\right)
$$

If $\operatorname{Jac}_{x^{*}}(S)$ is invertible then:

$$
g_{n} \underset{n \rightarrow+\infty}{\longrightarrow} \frac{1}{\sqrt{K}} \delta_{x^{*}} \quad \text { In the sense of distributions }
$$

Proof. It results from the theorem 1. In fact

$$
\operatorname{Hess}_{x^{*}}(g)=\left(\begin{array}{ccc}
\frac{\partial^{2} g}{\partial x_{1}^{2}}\left(x^{*}\right) & \ldots & \frac{\partial^{2} g}{\partial x_{1} \partial x_{d}}\left(x^{*}\right) \\
\vdots & \ddots & \vdots \\
\frac{\partial^{2} g}{\partial x_{1} \partial x_{d}}\left(x^{*}\right) & \ldots & \frac{\partial^{2} g}{\partial x_{d}^{2}}\left(x^{*}\right)
\end{array}\right)
$$

And for two integers $i$ and $j$ less or equal than $d$

$$
\frac{\partial^{2} g}{\partial x_{i} \partial x_{j}}\left(x^{*}\right)=\frac{\partial}{\partial x_{i}}\left(\frac{\partial}{\partial x_{j}}\left(f_{1}^{2}+\ldots+f_{d}^{2}\right)\right)_{x^{*}}=2 \frac{\partial}{\partial x_{i}}\left(f_{1} \frac{\partial f_{1}}{\partial x_{j}}+\ldots+f_{d} \frac{\partial f_{d}}{\partial x_{j}}\right)_{x^{*}}
$$

$f_{1}\left(x^{*}\right)=\ldots=f_{d}\left(x^{*}\right)=0$ then

$$
\frac{\partial^{2} g}{\partial x_{i} \partial x_{j}}\left(x^{*}\right)=2\left(\frac{\partial f_{1}}{\partial x_{i}} \frac{\partial f_{1}}{\partial x_{j}}+\ldots+\frac{\partial f_{d}}{\partial x_{i}} \frac{\partial f_{d}}{\partial x_{j}}\right)_{x^{*}}
$$

Therefore

$$
\operatorname{Hess}_{x^{*}}(g)=2\left({ }^{t} J a c_{x^{*}}\left(f_{1}\right) \cdot J a c_{x^{*}}\left(f_{1}\right)+\ldots+{ }^{t} J a c_{x^{*}}\left(f_{d}\right) . J a c_{x^{*}}\left(f_{d}\right)\right)
$$


$\forall x \in \mathbb{R}^{d}$ and $x \neq 0$

$$
{ }^{t} x . \operatorname{Hess}_{x^{*}}(g) . x=2 \sum_{i=1}^{d}\left(\left(J a c_{x^{*}}\left(f_{i}\right)\right) . x\right)^{2}
$$

As $J a c_{x^{*}}(S)$ is invertible then $\left({ }^{t} J a c_{x^{*}}\left(f_{1}\right), \ldots,{ }^{t} J a c_{x^{*}}\left(f_{d}\right)\right)$ is a basis of $\mathbb{R}^{d}$, therefore ${ }^{t} x . \operatorname{Hess}_{x^{*}}(g) \cdot x>0$ because if ${ }^{t} x \cdot \operatorname{Hess}_{x^{*}}(g) \cdot x=0 \Rightarrow\left(\operatorname{Jac}_{x^{*}}\left(f_{i}\right)\right) \cdot x=0 \forall i=1, \ldots, d$ then $x$ is orthogonal to all vectors of the basis thus $x$ is the zero vector. We conclude that $\operatorname{Hess}_{x^{*}}(g)$ is positive definite.

\section{Climbing the Dimensions Algorithm for Finding the Solution}

This section will present an algorithm to find the zero $x^{*}$ of the function $g$ which satisfies all conditions presented in the theorem 1.

We take into consideration the sequence of functions $g_{n}(x)=\mathbf{1}_{\Omega}(x)\left(n \sqrt{\frac{1}{2 \pi}}\right)^{d} e^{-n^{2} g(x)}$. Using theorem 1 we have:

$$
\lim _{n \rightarrow+\infty} \int_{\mathbb{R}^{d}} g_{n}(x) \phi(x) d \lambda(x)=\frac{1}{\sqrt{c}} \phi\left(x^{*}\right) \quad \forall \phi \in \mathcal{D}\left(\mathbb{R}^{d}\right)
$$

Where $c=\operatorname{det}\left(\operatorname{Hess}_{x^{*}}(g)\right)$.

We consider a several test functions for this algorithm, the idea is to trap the solution each time in a sphere to finish in the intersection of these spheres, where we will have in the end 2 points where one is the solution and the other is outside the domain.

We will consider the following test function from the space $\mathcal{D}\left(\mathbb{R}^{d}\right)$ :

$$
\phi_{r, a}(x)=\left\{\begin{array}{l}
e^{\frac{-1}{r^{2}-\|x-a\|^{2}}} \text { if } x \in B(a, r) \\
0 \text { otherwise }
\end{array}\right.
$$

For each test function $\phi_{r, a}$ and for each $n \in \mathbb{N}^{*}$ we call $S_{\phi_{r, a}}\left(a, \rho_{n}\right)$ the sphere of center $a$ and of radius $\rho_{n}$ where $\rho_{n}=\sqrt{r^{2}-\frac{1}{\operatorname{Ln}\left(y_{n}\right)}}$ and $y_{n}=\frac{\int_{\mathbb{d}} g_{n} \phi_{r, a} d \lambda}{\int_{\mathbb{R} d} g_{n} \phi_{r, d}^{2} d \lambda}$.

Besides if we have 2 spheres $S(\xi, r) \subset \mathbb{R}^{d}$ and $S(\eta, R) \subset \mathbb{R}^{d}$ of centers respectively $\xi$ and $\eta$, of radius respectively $r$ and $R$ such that $\xi \neq \eta$ and $S(\xi, r) \cap S(\eta, R) \neq \varnothing$, then $S(\xi, r) \cap S(\eta, R)$ is a sphere in $\mathbb{R}^{d-1}$ with a radius equal to $\sqrt{r^{2}-\left(\frac{L^{2}+r^{2}-R^{2}}{2 L}\right)^{2}}$ and with a center $\chi$ belongs to the line $(\xi, \eta)$ such that $\chi=x_{1} \frac{\eta-\xi}{L}+\xi$, where $L=\|\eta-\xi\|$ and $x_{1}=\frac{L^{2}+r^{2}-R^{2}}{2 L}$.

Now we begin the algorithm, let $n \in \mathbb{N}^{*}$ a bit big, let $a \in \Omega$ and $R^{*}>0$ such that $\Omega \subset B\left(a, R^{*}\right)$. Let $\left(e_{1}, \ldots, e_{d}\right)$ the orthonormal basis of $\mathbb{R}^{d}$, we choose $\eta_{0}$ such that $\eta_{0}-a=K e_{1}$ where $K>R^{*}$.

We take the test function $\phi_{r_{0}, \eta_{0}}$ such that $\Omega \subset B\left(\eta_{0}, r_{0}\right)$ we obtain then the sphere $S_{\phi_{r_{0}, \eta_{0}}}\left(\eta_{0}, \rho_{0 n}\right)$.

We choose $\chi_{0}$ such that $\chi_{0}-\eta_{0}=\rho_{0 n} e_{2} \Rightarrow\left\|\chi_{0}-\eta_{0}\right\|=\rho_{0 n} \Rightarrow \chi_{0} \in S_{\phi_{r_{0}, \eta_{0}}}\left(\eta_{0}, \rho_{0 n}\right)$. We take also a test function $\phi_{r_{1}, \chi_{0}}$ such that $\Omega \subset B\left(\chi_{0}, r_{1}\right)$ we obtain then the sphere $S_{\phi_{r_{1} \chi_{0}}}\left(\chi_{0}, \rho_{1 n}\right)$.

Next we consider the intersection of the 2 spheres:

$$
S_{\phi_{r_{0}, \eta_{0}}}\left(\eta_{0}, \rho_{0 n}\right) \cap S_{\phi_{r_{1}, \chi_{0}}}\left(\chi_{0}, \rho_{1 n}\right)=S\left(\eta_{1}, R_{1}\right) \subset \mathbb{R}^{d-1}
$$

Where $\eta_{1}$ and $R_{1}$ are easily obtained from before and $\eta_{1} \in\left(\eta_{0}, \chi_{0}\right)$.

Next we choose $\chi_{1}$ such that $\chi_{1}-\eta_{1}=R_{1} e_{3} \Rightarrow\left\|\chi_{1}-\eta_{1}\right\|=R_{1} \Rightarrow \chi_{1} \in S\left(\eta_{1}, R_{1}\right)$. Then

$$
\left\{\begin{array}{l}
\left\|\chi_{1}-\eta_{1}\right\|=R_{1} \\
<\chi_{1}-\eta_{1} \mid \chi_{0}-\eta_{0}>=0
\end{array}\right.
$$

The symbol $<. \mid .>$ denotes the scalar product in $\mathbb{R}^{d}$.

We take also a test function $\phi_{r_{2}, \chi_{1}}$ such that $\Omega \subset B\left(\chi_{1}, r_{2}\right)$ we obtain then the sphere $S_{\phi_{r_{2} \chi_{1}}}\left(\chi_{1}, \rho_{2 n}\right)$

We consider the intersection of the 2 spheres:

$$
S\left(\eta_{1}, R_{1}\right) \cap S_{\phi_{r_{2} \times 1}}\left(\chi_{1}, \rho_{2 n}\right)=S\left(\eta_{2}, R_{2}\right) \subset \mathbb{R}^{d-2}
$$


With $\eta_{2} \in\left(\eta_{1}, \chi_{1}\right)$

By recurrence for $1 \leq k \leq d-2$ we choose $\chi_{k}$ such that $\chi_{k}-\eta_{k}=R_{k} e_{k+2} \Rightarrow \chi_{k}=\eta_{k}+R_{k} e_{k+2} \Rightarrow \chi_{k} \in S\left(\eta_{k}, R_{k}\right)$. Then

$$
\left\{\begin{array}{l}
\left\|\chi_{k}-\eta_{k}\right\|=R_{k} \\
<\chi_{k}-\eta_{k} \mid \chi_{k-1}-\eta_{k-1}>=0 \\
\vdots \\
<\chi_{k}-\eta_{k} \mid \chi_{0}-\eta_{0}>=0
\end{array}\right.
$$

Where $R_{k}$ and $\eta_{k}$ are calculated from the intersection:

$$
S\left(\eta_{k-1}, R_{k-1}\right) \cap S_{\phi_{r_{k}, \chi_{k-1}}}\left(\chi_{k-1}, \rho_{k n}\right)=S\left(\eta_{k}, R_{k}\right) \subset \mathbb{R}^{d-k}
$$

Where $S_{\phi_{r_{k}, \chi_{k-1}}}\left(\chi_{k-1}, \rho_{k n}\right)$ corresponds to the test function $\phi_{r_{k}, \chi_{k-1}}$ such that $\Omega \subset B\left(\chi_{k-1}, r_{k}\right)$.

In conclusion $\forall 0 \leq k \leq d-3$

$$
\left\{\begin{aligned}
\chi_{k+1} & =\eta_{k+1}+R_{k+1} e_{k+3} \\
R_{k+1} & =\sqrt{R_{k}^{2}-\left(\frac{2 R_{k}^{2}-\rho_{(k+1) n}^{2}}{2 R_{k}}\right)^{2}} \\
\eta_{k+1} & =\left(\frac{2 R_{k}^{2}-\rho_{(k+1) n}^{2}}{2 R_{k}^{2}}\right)\left(\chi_{k}-\eta_{k}\right)+\eta_{k}
\end{aligned}\right.
$$

With $R_{0}=\rho_{0 n}$ and where $\chi_{0}=\eta_{0}+\rho_{0 n} e_{2}$ and $\rho_{(k+1) n}$ corresponds to the test function $\phi_{r_{k+1}, \chi_{k}}$ such that $\Omega \subset B\left(\chi_{k}, r_{k+1}\right)$.

Otherwise $\eta_{d-1}-\eta_{d-2}$ is collinear to $e_{d}, \eta_{0}-a$ is collinear to $e_{1}$ and $\forall 1 \leq i \leq d-2, \eta_{i}-\eta_{i-1}$ is collinear to $e_{i+1}$. Therefore $\forall 1 \leq k \leq d-1$

$$
\begin{gathered}
\left\|\eta_{k}-a\right\|^{2}=\left\|\left(\eta_{k}-\eta_{k-1}\right)+\ldots+\left(\eta_{1}-\eta_{0}\right)+\left(\eta_{0}-a\right)\right\|^{2} \\
\Rightarrow\left\|\eta_{k}-a\right\|^{2}=\left\|\eta_{k}-\eta_{k-1}\right\|^{2}+\ldots+\left\|\eta_{0}-a\right\|^{2}
\end{gathered}
$$

$\left\|\eta_{0}-a\right\|>R^{*} \Rightarrow\left\|\eta_{k}-a\right\|>R^{*}$. Therefore $\eta_{k} \notin \Omega$.

When $k=d-3$ then $\chi_{d-2}-\eta_{d-2}=R_{d-2} e_{d} \Rightarrow S\left(\eta_{d-2}, R_{d-2}\right) \subset \mathbb{R}^{2} . \eta_{d-1}$ and $R_{d-1}$ corresponds to the intersection $S\left(\eta_{d-2}, R_{d-2}\right) \cap S_{\phi_{r_{d-1}, \chi_{d-2}}}\left(\chi_{d-2}, \rho_{(d-1) n}\right)$ which is a two points $H_{1}$ and $H_{2} . H_{1}=-R_{d-1} e_{1}+\eta_{d-1}$ and $H_{2}=R_{d-1} e_{1}+\eta_{d-1}$. Therefore

$$
\left\|H_{2}-a\right\|^{2}=\left\|H_{2}-\eta_{d-1}\right\|^{2}+\left\|\eta_{d-1}-a\right\|^{2}+2<H_{2}-\eta_{d-1} \mid \eta_{d-1}-a>
$$

We have $H_{2}-\eta_{d-1}=R_{d-1} e_{1}, \eta_{d-1}-a=\left(\eta_{d-1}-\eta_{d-2}\right)+\ldots+\left(\eta_{1}-\eta_{0}\right)+\left(\eta_{0}-a\right)$, and $\eta_{0}-a$ is collinear to $e_{1}, \eta_{0}-a=K e_{1}$ thus:

$$
<H_{2}-\eta_{d-1}\left|\eta_{d-1}-a>=<R_{d-1} e_{1}\right| K e_{1}>=K R_{d-1}>0
$$

Therefore

$$
\begin{aligned}
\left\|H_{2}-a\right\|^{2} & \geq\left\|\eta_{d-1}-a\right\|^{2}>\left(R^{*}\right)^{2} \\
& \Rightarrow H_{2} \notin \Omega
\end{aligned}
$$

In conclusion we consider the sequence $x_{n}=H_{1}=-R_{d-1} e_{1}+\eta_{d-1}$.

Let $r>0$ and $c \in \mathbb{R}^{d}$, let $\phi(x)=e^{\frac{-1}{r^{2}-\|x-c\|^{2}}} \mathbf{1}_{B(c, r)}(x)$, we take two sequences $r_{n}>0$ and $c_{n} \in \mathbb{R}^{d}$ such that $r_{n} \underset{n \rightarrow+\infty}{\longrightarrow} r$ and $c_{n} \underset{n \rightarrow+\infty}{\longrightarrow} c$ therefore:

$$
\phi_{n}(x)=e^{\frac{-1}{r_{n}^{2}-\left\|x-c_{n}\right\|^{2}}} \mathbf{1}_{B\left(c_{n}, r_{n}\right)}(x) \underset{n \rightarrow+\infty}{\longrightarrow} \phi(x) \forall x \in \mathbb{R}^{d}
$$

If we take the same notations as in the proof of the theorem 1. then:

$$
<g_{n}, \phi_{n}>=\int_{\mathbb{R}^{d}}\left(G_{n}(x)-F_{n}(x)\right) \phi_{n}\left(O^{-1} x+x^{*}\right) d \lambda(x)+\int_{\mathbb{R}^{d}} F_{n}(x) \phi_{n}\left(O^{-1} x+x^{*}\right) d \lambda(x)
$$

And

$$
\phi_{n}\left(O^{-1} x+x^{*}\right)=\left(\phi_{n}\left(O^{-1} x+x^{*}\right)-\phi\left(O^{-1} x+x^{*}\right)\right)+\phi\left(O^{-1} x+x^{*}\right)
$$

As $\phi_{n}$ and $\phi$ are bounded and by using the Dominated Convergence theorem, we obtain

$$
<g_{n}, \phi_{n}>\underset{n \rightarrow+\infty}{\longrightarrow} \frac{1}{\sqrt{\operatorname{det}\left(\operatorname{Hess}_{x^{*}}(g)\right)}} \phi\left(x^{*}\right)
$$


If $x^{*} \in B(c, r)$ then

$$
\frac{<g_{n}, \phi_{n}>}{<g_{n}, \phi_{n}^{2}>} \underset{n \rightarrow+\infty}{\longrightarrow} e^{\frac{1}{r^{2}-\| x^{*}-\left.c\right|^{2}}}
$$

Let $\xi_{n}=\frac{<g_{n}, \phi_{n}>}{<g_{n}, \phi_{n}^{2}>}$ and

$$
H_{n}=\sqrt{r^{2}-\frac{1}{\operatorname{Ln}\left(\xi_{n}\right)}}
$$

Therefore

$$
\begin{gathered}
\lim _{n \rightarrow+\infty} H_{n}=\left\|x^{*}-c\right\| \\
\Rightarrow\left\|x^{*}-c\right\|=\lim _{n \rightarrow+\infty} H_{n}=H
\end{gathered}
$$

Thus $x^{*} \in S(c, H)$. Back to the algorithm we have: $\forall 0 \leq k \leq d-3$

$$
\left\{\begin{aligned}
\chi_{k+1} & =\eta_{k+1}+R_{k+1} e_{k+3} \\
R_{k+1} & =\sqrt{R_{k}^{2}-\left(\frac{2 R_{k}^{2}-\rho_{(k+1)}^{2}}{2 R_{k}}\right)^{2}} \\
\eta_{k+1} & =\left(\frac{2 R_{k}^{2}-\rho_{(k+1)}^{2}}{2 R_{k}^{2}}\right)\left(\chi_{k}-\eta_{k}\right)+\eta_{k}
\end{aligned}\right.
$$

With $R_{0}=\rho_{0}$ and $\chi_{0}=\eta_{0}+\rho_{0} e_{2}$. Where

$$
\left\{\begin{aligned}
\rho_{0} & =\lim _{n \rightarrow+\infty} \rho_{0 n} \\
\rho_{k} & =\lim _{n \rightarrow+\infty} \rho_{k n} \forall 1 \leq k \leq d-1 \\
R_{k} & =\lim _{n \rightarrow+\infty}\left(R_{k}\right)_{n} \forall 1 \leq k \leq d-2 \\
\eta_{k} & =\lim _{n \rightarrow+\infty}\left(\eta_{k}\right)_{n} \forall 1 \leq k \leq d-2 \\
\chi_{k} & =\lim _{n \rightarrow+\infty}\left(\chi_{k}\right)_{n} \forall 1 \leq k \leq d-2
\end{aligned}\right.
$$

Therefore $x^{*} \in S\left(\eta_{0}, R_{0}\right)$ and $x^{*} \in S\left(\chi_{0}, \rho_{1}\right) \Rightarrow x^{*} \in S\left(\eta_{0}, R_{0}\right) \cap S\left(\chi_{0}, \rho_{1}\right)=S\left(\eta_{1}, R_{1}\right)$. We repeat the procedure to obtain:

$$
x^{*} \in S\left(\eta_{d-2}, R_{d-2}\right) \cap S\left(\chi_{d-2}, \rho_{d-1}\right)
$$

Therefore $x^{*}=-R_{d-1} e_{1}+\eta_{d-1}$ or $x^{*}=R_{d-1} e_{1}+\eta_{d-1}$. Otherwise we have $\left(H_{2}\right)_{n}=\left(R_{d-1} e_{1}+\eta_{d-1}\right)_{n} \notin \Omega$ thus $x^{*}=$ $-R_{d-1} e_{1}+\eta_{d-1}$. We conclude that:

$$
\lim _{n \rightarrow+\infty} x_{n}=x^{*}
$$

In other words we can obtain an approximation of the solution $x^{*}$ by only computing $x_{n}$ for a big value of $n$.

Otherwise we can use this approximation for another algorithm for solving nonlinear systems of equations.

\subsection{Examples}

\subsubsection{Example in 2D}

Let us take a simple system of equations:

$$
(S):\left\{\begin{array}{l}
e^{x+y}=1 \\
x-y=0
\end{array}\right.
$$

The exact solution of this system is: $(x, y)=(0,0)$.

In order to apply our algorithm we consider the function $g(x, y)=\left(e^{x+y}-1\right)^{2}+(x-y)^{2}$ and its corresponding sequence of functions $g_{n}(x, y)=\mathbf{1}_{B((0,0), 2)}(x, y)\left(\frac{n}{\sqrt{2 \pi}}\right)^{2} e^{-n^{2} g(x, y)}$.

Let $\operatorname{Jac}_{(x, y)}(S)$ be the Jacobian of the system $(S)$ at $(x, y)$ thus:

$$
\operatorname{Jac}_{(x, y)}(S)=\left(\begin{array}{ll}
\frac{\partial f_{1}}{\partial x}(x, y) & \frac{\partial f_{1}}{\partial y}(x, y) \\
\frac{\partial f_{2}}{\partial x}(x, y) & \frac{\partial f_{2}}{\partial y}(x, y)
\end{array}\right)=\left(\begin{array}{cc}
e^{x+y} & e^{x+y} \\
1 & -1
\end{array}\right)
$$

Where $f_{1}(x, y)=e^{x+y}-1$ and $f_{2}(x, y)=x-y$.

The determinant of $\operatorname{Jac}_{(x, y)}(S)$ is equal to: $\operatorname{det}\left(\operatorname{Jac}_{(x, y)}(S)\right)=-2 e^{x+y} \neq 0$. Thus the function $g$ satisfies all conditions presented in the theorem 1. 
First we take the test function:

$$
\phi_{r_{0}, \eta_{0}}(z)=\left\{\begin{array}{l}
e^{\frac{-1}{r_{0}-\left\|z-\eta_{0}\right\|^{2}}} \quad \text { if } z \in B\left(\eta_{0}, r_{0}\right) \\
0 \text { otherwise }
\end{array}\right.
$$

Where $\eta_{0}=(3,0)$ and $r_{0}=6$.

Therefore by taking $n=7$ we obtain the sphere $S_{\phi_{r_{0}, \eta_{0}}}\left(\eta_{0}, R_{0}\right)$ where $R_{0}=3.011$, then we choose $\chi_{0}=\eta_{0}+R_{0} e_{2}=$ $(3,3.011)$. By choosing the second test function:

$$
\phi_{r_{1}, \chi_{0}}(z)=\left\{\begin{array}{l}
e^{\frac{-1}{r_{1}-\left\|z-\chi_{0}\right\|^{2}}} \quad \text { if } z \in B\left(\chi_{0}, r_{1}\right) \\
0 \text { otherwise }
\end{array}\right.
$$

Where $r_{1}=7$, we obtain then the sphere $S_{\phi_{r_{1}, x_{0}}}\left(\chi_{0}, \rho_{1}\right)$ where $\rho_{1}=4.26$. Then the approximate solution $z_{7}$ is at the intersection of $S_{\phi_{r_{0}, \eta_{0}}}\left(\eta_{0}, R_{0}\right) \cap S_{\phi_{r_{1}, \chi_{0}}}\left(\chi_{0}, \rho_{1}\right)$, thus

$$
z_{7}=-R_{1} e_{1}+\eta_{1}
$$

Where $R_{1}=\sqrt{R_{0}^{2}-\left(\frac{2 R_{0}^{2}-\rho_{1}^{2}}{2 R_{0}}\right)^{2}}=3.01$ and $\eta_{1}=\left(\frac{2 R_{0}^{2}-\rho_{1}^{2}}{2 R_{0}^{2}}\right)\left(\chi_{0}-\eta_{0}\right)+\eta_{0}=(3,-0.01)$

We conclude that $z_{7}=(-0.01,-0.01)$.

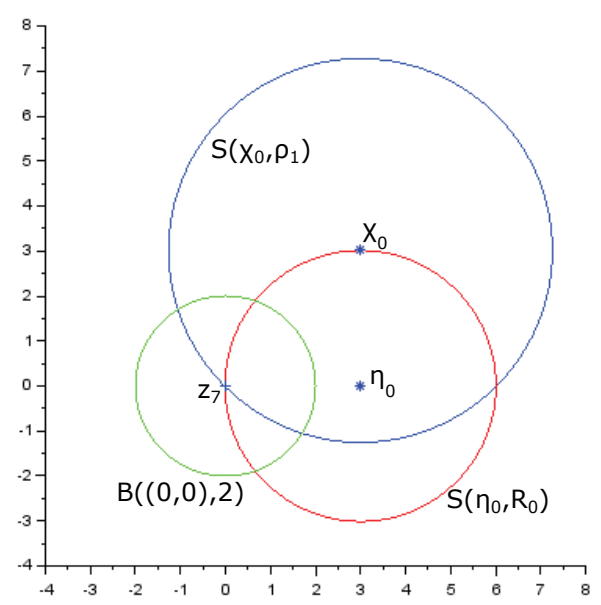

Figure 2. The approximate solution $z_{7}$

\subsubsection{Example in 3D}

Let us take a simple system of equations:

$$
(S):\left\{\begin{array}{l}
\operatorname{Ln}(x)=\operatorname{Ln}(2) \\
\operatorname{Ln}(y)=\operatorname{Ln}(2) \\
\operatorname{Ln}(z)=\operatorname{Ln}(2)
\end{array}\right.
$$

The exact solution of this system is: $(x, y, z)=(2,2,2)$.

In order to apply our algorithm we will search for a solution in $\Omega=B((3,3,3), 2)$, thus we take the function:

$$
g(x, y, z)=(\operatorname{Ln}(x)-\operatorname{Ln}(2))^{2}+(\operatorname{Ln}(y)-\operatorname{Ln}(2))^{2}+(\operatorname{Ln}(z)-\operatorname{Ln}(2))^{2}
$$

and its corresponding sequence of functions $g_{n}(x, y, z)=\mathbf{1}_{B((3,3,3), 2)}(x, y, z)\left(\frac{n}{\sqrt{2 \pi}}\right)^{3} e^{-n^{2} g(x, y, z)}$. Let $\operatorname{Jac}_{(x, y, z)}(S)$ be the Jacobian of the system $(S)$ at $(x, y, z)$ thus:

$$
\operatorname{Jac}_{(x, y, z)}(S)=\left(\begin{array}{ccc}
\frac{1}{x} & 0 & 0 \\
0 & \frac{1}{y} & 0 \\
0 & 0 & \frac{1}{z}
\end{array}\right)
$$


The determinant of $\operatorname{Jac}_{(x, y, z)}(S)$ is equal to: $\operatorname{det}\left(\operatorname{Jac}_{(x, y, z)}(S)\right)=\frac{1}{x y z} \neq 0 \forall(x, y, z) \in \Omega$. Thus the function $g$ satisfies all conditions presented in the theorem 1 .

First we take the test function:

$$
\phi_{r_{0}, \eta_{0}}(w)=\left\{\begin{array}{l}
e^{\frac{-1}{r_{0}-\left\|w-\eta_{0}\right\|^{2}}} \text { if } w \in B\left(\eta_{0}, r_{0}\right) \\
0 \text { otherwise }
\end{array}\right.
$$

Where $\eta_{0}=(6,3,3)$ and $r_{0}=6$.

Therefore by taking $n=9$ we obtain the sphere $S_{\phi_{0,}, \eta_{0}}\left(\eta_{0}, R_{0}\right)$ where $R_{0}=4.23$, then we choose $\chi_{0}=\eta_{0}+R_{0} e_{2}=$ $(6,7.23,3)$. By choosing the second test function:

$$
\phi_{r_{1}, \chi_{0}}(z)=\left\{\begin{array}{l}
e^{\frac{-1}{r_{1}^{2}-\left\|z-\chi_{0}\right\|^{2}}} \text { if } z \in B\left(\chi_{0}, r_{1}\right) \\
0 \text { otherwise }
\end{array}\right.
$$

Where $r_{1}=8$, we obtain then the sphere $S_{\phi_{r_{1}, \chi_{0}}}\left(\chi_{0}, \rho_{1}\right)$ where $\rho_{1}=6.64$. Then we consider the intersection of $S_{\phi_{r_{0}, \eta_{0}}}\left(\eta_{0}, R_{0}\right) \cap$ $S_{\phi_{r_{1}, 0}}\left(\chi_{0}, \rho_{1}\right)=S\left(\eta_{1}, R_{1}\right)$, which is a circle.

Where $R_{1}=\sqrt{R_{0}^{2}-\left(\frac{2 R_{0}^{2}-\rho_{1}^{2}}{2 R_{0}}\right)^{2}}=4.11$ and $\eta_{1}=\left(\frac{2 R_{0}^{2}-\rho_{1}^{2}}{2 R_{0}^{2}}\right)\left(\chi_{0}-\eta_{0}\right)+\eta_{0}=(6,2.01,3)$.

We choose $\chi_{1}=\eta_{1}+R_{1} e_{3}=(6,2.01,7.11)$. By choosing the third test function:

$$
\phi_{r_{2}, \chi_{1}}(z)=\left\{\begin{array}{l}
e^{\frac{-1}{r_{2}-\left\|z-\chi_{1}\right\|^{2}}} \text { if } z \in B\left(\chi_{1}, r_{2}\right) \\
0 \text { otherwise }
\end{array}\right.
$$

Where $r_{2}=9$, we obtain then the sphere $S_{\phi_{r_{2} \times 1}}\left(\chi_{1}, \rho_{2}\right)$ where $\rho_{2}=6.47$. Then the approximate solution $z_{9}$ is at the intersection of $S_{\phi_{r_{2} \chi_{1}}}\left(\chi_{1}, \rho_{2}\right) \cap S\left(\eta_{1}, R_{1}\right)$, thus

$$
z_{9}=-R_{2} e_{1}+\eta_{2}
$$

Where $R_{2}=\sqrt{R_{1}^{2}-\left(\frac{2 R_{1}^{2}-\rho_{2}^{2}}{2 R_{1}}\right)^{2}}=3.99$ and $\eta_{2}=\left(\frac{2 R_{1}^{2}-\rho_{2}^{2}}{2 R_{1}^{2}}\right)\left(\chi_{1}-\eta_{1}\right)+\eta_{1}=(6,2.01,2.02)$.

We conclude that $z_{9}=(2.01,2.01,2.02)$.

\section{Conclusion}

The method presented above is a generalization of (Houssein, 2018) and it demands only to make an integration in order to find the solution of the nonlinear equation, it presents an advantages compared to some other methods. Otherwise the approximate solution can be used as a starting point for iterative methods.

\section{References}

Houssein, H. (2018). An Algorithm For Solving Nonlinear Equations Using Distributions. Journal of Mathematics Research, 10(5), 37-44. https://doi.org/10.5539/jmr.v10n5p37

Schwartz, L. (1963). Some applications of the theory of distributions. Lectures on Modern Mathematics, I, New York: Wiley, 23-58.

Schwartz, L. (1966). Théorie des distributions. Paris: Hermann.

\section{Copyrights}

Copyright for this article is retained by the author(s), with first publication rights granted to the journal.

This is an open-access article distributed under the terms and conditions of the Creative Commons Attribution license (http://creativecommons.org/licenses/by/4.0/). 